\title{
Aut liberi aut libri? Arbeitsbedingungen und - zufriedenheit des religionswissenschaftlichen Nachwuchses in Deutschland
}

Ergebnisse einer Umfrage des Arbeitskreises Mittelbau und Nachwuchs (AKMN) der Deutschen Vereinigung für Religionswissenschaft (DVRW)

Veronika Eufinger, Ramona Jelinek-Menke and Anna Neumaier

\section{(2) OpenEdition}

\section{Journals}

Electronic version

URL: http://journals.openedition.org/zjr/673

DOI: $10.4000 /$ zjr.673

ISSN: $1862-5886$

\section{Publisher}

Deutsche Vereinigung für Religionswissenschaft

Electronic reference

Veronika Eufinger, Ramona Jelinek-Menke und Anna Neumaier, « Aut liberi aut libri?

Arbeitsbedingungen und -zufriedenheit des religionswissenschaftlichen Nachwuchses in Deutschland ", Zeitschrift für junge Religionswissenschaft [Online], 11 | 2016, Online erschienen am: 22 November 2016, abgerufen am 02 Mai 2019. URL : http://journals.openedition.org/zjr/673 ; DOI : 10.4000/zjr.673

This text was automatically generated on 2 mai 2019.

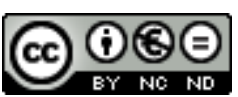

Dieses Werk ist lizenziert unter einer Creative Commons Namensnennung - Nicht-kommerziell - Keine Bearbeitung 3.0 Deutschland Lizenz. 


\section{Aut liberi aut libri? Arbeitsbedingungen und - zufriedenheit des religionswissenschaftlichen Nachwuchses in Deutschland}

Ergebnisse einer Umfrage des Arbeitskreises Mittelbau und Nachwuchs (AKMN) der Deutschen Vereinigung für Religionswissenschaft (DVRW)

\section{AUTHOR'S NOTE}

Dieser Artikel wird ebenso in der Ausgabe 24 (2) der Zeitschrift für Religionswissenschaft (2016) veröffentlicht. Wir danken den Herausgeber*innen beider Zeitschriften für die Möglichkeit, mit der parallelen Veröffentlichung den Artikel einer breiten Öffentlichkeit zugänglich zu machen.

\section{Einordnung}

"Denn es ist außerordentlich gewagt für einen jungen Gelehrten, der keinerlei Vermögen hat, überhaupt den Bedingungen der akademischen Laufbahn sich auszusetzen. Er muss es mindestens eine Anzahl Jahre aushalten können, ohne irgendwie zu wissen, ob er nachher die Chancen hat, einzurücken in eine Stellung, die für den Unterhalt ausreicht (Weber 1992 [1917/19], 72).«

Man könnte versucht sein, Unsicherheiten mit Blick auf die Planung einer wissenschaftlichen Karriere für einen rezenten Nebeneffekt allgemeiner Neoliberalisierungstendenzen eines drittmittelorientierten Wissenschaftsbetriebes $\mathrm{zu}$ halten. Doch das angeführte, in den Kreisen der Betroffenen schon berühmt-berüchtigte 
Zitat stammt eben nicht aus dem im Januar 2016 veröffentlichten Imboden-Bericht oder der Wissenschaftssparte der ZEIT, sondern verweist vielmehr auf die allzu bequeme Tradition dieser Problemlage: Vor fast einem Jahrhundert diagnostiziert Max Weber, dass »bei uns die Laufbahn eines Mannes der Wissenschaft im Ganzen auf plutokratischen Voraussetzungen aufgebaut ist (Weber 1992, 71-72.), « und fährt mit dem obenstehenden Hinweis auf die damit verbundenen Risiken fort. ${ }^{1}$

2 Angesichts dieser Diagnose erscheint es umso erstaunlicher, dass sich (außer der explizierten geschlechtlichen Zuordnung) die Zustände, das Narrativ und die darum kreisenden Diskussionen bis heute kaum verbessert haben: Die akademische Laufbahn ist weiterhin hochprekär; sie besteht aus langen Jahren voller Unsicherheit, an die sich endlich die ersehnte entfristete Stelle oder - wahrscheinlicher - eine späte Arbeitslosigkeit anschließen. Dies kannibalisiert letztlich auch die Wissenschaft selbst: Begabte Akademiker*innen verlassen die Universität für andere Jobs mit klareren Perspektiven oder einer günstigeren Work-Life-Balance oder kehren zumindest dem Wissenschaftsstandort Deutschland den Rücken, um ihre Zukunft in Hochschulsystemen anderer Länder zu suchen, die ihnen verlockendere Arbeitsbedingungen bieten. Diese Sorge schlägt sich auch im Imboden-Bericht nieder:

»Die internationale Attraktivität und Wettbewerbsfähigkeit des deutschen Hochschulsektors hängt ganz erheblich davon ab, ob es gelingt, begabte junge Menschen als wissenschaftlichen Nachwuchs zu rekrutieren, zu qualifizieren und in das System zu integrieren. Eine wichtige Voraussetzung für Spitzenforschung ist ein effizientes und transparentes Nachwuchssystem, das den Leistungsfähigsten eine planbare Chance für eine akademische Karriere einräumt (Internationale Expertenkommission Exzellenzinitiative 2016, 25).《

Abgesehen von dem systemischen Interesse mag man aber auch die Frage nach der Perspektive der betroffenen akademischen Mitarbeiter*innen stellen: Wie blicken sie auf diese Arbeitsverhältnisse? Wie und mit welchen Aufgaben sind sie tatsächlich beschäftigt? An welcher Stelle liegen besondere Problemlagen? Inwiefern sind Universitäten dennoch attraktive Arbeitgeber? Obgleich es keine breite Datenlage über wissenschaftliche Angestellte gibt, haben zuletzt einige Erhebungen diese Fragen in den Blick genommen. So entstand im Auftrag der Dienstleistungsgewerkschaft ver.di 2009 eine Untersuchung zur Lage der Promovierenden mit dem Untertitel "Zwischen Karriereaussichten und Abbruchtendenzen«. Der verwendete Fragebogen deckte Themenfelder rund um die berufliche Situation der Nachwuchswissenschaftler*innen, die subjektive Bewertung dieser Situation, die Einschätzung der eigenen beruflichen Zukunft sowie das Verhältnis zu den Gewerkschaften ab. Die Zuordnung der Fachrichtungen erfolgte anhand von insgesamt 19 Auswahlmöglichkeiten (Grühn et al 2009, 7-11.)2. Unter dem Titel »Promotionen im Fokus« erstellte das Hochschul-Informations-System (HIS) 2012 auf Basis einer Online-Panelbefragung eine vergleichende Studie zu den Promotionsund Arbeitsbedingungen von Doktorand*innen in Deutschland (Jaksztat, Preßler und Briedis 2012). Die thematischen Schwerpunkte lagen auf der Betreuungssituation und der wissenschaftlichen Qualität im Rahmen des Promotionsverfahrens, den Arbeitsbedingungen im wissenschaftlichen Betrieb sowie den privaten Lebensverhältnissen hinsichtlich Partnerschaft und Familie. Die Ergebnisse wurden dabei nach den fünf Fächergruppen Geisteswissenschaften, Erziehungswissenschaften/ Psychologie, Rechts-/Wirtschafts-/Sozialwissenschaften, Mathematik/ Naturwissenschaften und Ingenieurswissenschaften differenziert (Jaksztat, Preßler und Briedis 2012, 1-4.). ${ }^{3}$ Der Fokus des aktuellen »Bundesberichts Wissenschaftlicher 
Nachwuchs« von 2013 lag auf den Arbeitsverhältnissen, Beschäftigungsformen und dem weiteren Karriereweg von Promovierenden sowie den beruflichen Perspektiven im außeruniversitären wissenschaftlichen Bereich. Die Kennwerte sind überwiegend struktureller Art, subjektive Einschätzungen Betroffener bilden nur einen kleinen Anteil. Es findet eine unterschiedlich starke Differenzierung anhand der Promotionsarten, Fächergruppen (zwischen fünf und zehn) und weiterer Kriterien statt (Konsortium Bundesbericht Wissenschaftlicher Nachwuchs 2013, 4-21). ${ }^{4}$

4 Die Unterscheidung der Ergebnisse mit Blick auf die akademischen Fächer in den Studien zur Situation des wissenschaftlichen Nachwuchses ist damit eher grober Natur. Religionswissenschaft als Fach wird dabei nicht getrennt ausgewiesen. Das facettenreiche fachliche Profil der Religionswissenschaft erschwert dabei auch umgekehrt die Einordnung in standardisierte Kategorien wie Geistes-, Kultur-, Sozialwissenschaften, Theologie oder »andere Fachrichtungen«. Entsprechend unmöglich ist es, aus den vorliegenden Studien spezifische Schlüsse für unser Fach zu ziehen.

5 Aus der Initiative von Mitgliedern des »Arbeitskreises Mittelbau und Nachwuchs" (AKMN) der »Deutschen Vereinigung für Religionswissenschaft« (DVRW) entstand daher das Vorhaben, eine Erhebung unter religionswissenschaftlich arbeitenden Akademiker*innen durchzuführen, die sich - hauptsächlich und erstmals - der Beschäftigungssituation und -zufriedenheit des religionswissenschaftlichen 'Nachwuchses (d.h. dem akademischen Mittelbau sowie, in Grenzen, extern Promovierenden und Habilitierenden) des deutschsprachigen Raumes widmet. ${ }^{5}$ Zentrale Ergebnisse dieser Studie möchten wir hier im Folgenden präsentieren. ${ }^{6}$ Nach einer Vorstellung des Erhebungsinstrumentes sowie einer allgemeinen Beschreibung der Erhebungsteilnehmer*innen und ihrer Beschäftigungssituation werden wir unser Augenmerk auf einige ausgewählte Themenbereiche legen: nämlich die (Un)Zufriedenheit mit Rahmenbedingungen und Perspektiven des Arbeitsverhältnisses, Belastungen am Arbeitsplatz und Gesundheit, Verhältnis zu Betreuer*innen und Kolleg*innen sowie Diskriminierung mit Blick auf Geschlecht und Familie. Die Befunde zu diesen Aspekten werden dabei auch, soweit möglich, mit Befunden anderer, etwa fächerübergreifender Studien, verglichen. ${ }^{7}$ Den Beitrag beschließen einige zusammenfassende Überlegungen und Handlungsempfehlungen, mit denen wir auch eine Diskussion im Fach über die Arbeitsbedingungen und die Beschäftigungsverhältnisse von (Religions-)Wissenschaftler*innen anzuregen hoffen.

\section{Zur Erhebung}

6 Erstmals wurde der Wunsch nach einer Erhebung unter den Promovierenden und Habilitierenden der deutschsprachigen Religionswissenschaft auf der Sitzung des Arbeitskreises "Mittelbau und Nachwuchs" der DVRW im Rahmen der DVRW-Tagung in Göttingen 2013 geäußert. Dies war verbunden mit dem Ziel, einen konkreten Eindruck ihrer Anstellungs- und Arbeitssituation zu erhalten. Nach intensiven Diskussionen im Kreis der AK-Mitglieder und -sprecher*innen wurde schließlich ein Fragebogen erstellt, der sich in weiten Teilen an der Umfrage der Mittelbauinitiative Universität Leipzig (MULE) zur Beschäftigungssituation des akademischen Mittelbaus an der Universität Leipzig orientierte (Mittelbauinitiative Universität Leipzig [MULE] 2015), ${ }^{8}$ die etwa zur gleichen Zeit wie die AKMN-Befragung entstand. Der Leipziger Fragebogen selbst beruhte wiederum auf einer Umfrage einer Dresdner Mittelbauinitiative bzw. modifizierte sie. 
Solche Rückgriffe und gegenseitigen Bezüge ermöglichen es, die Umfrageergebnisse von einzelnen Universitäten oder aus einzelnen Fächern in Bezug zur Gesamtsituation an deutschen Hochschulen zu setzen. Im Einzelnen wurden die Fragen speziell für die Zielgruppe der Religionswissenschaftler*innen angepasst.

Der erarbeitete und verwendete Fragebogen gliederte sich in fünf Blöcke. Es wurden sowohl die (vertrags-)rechtliche Stellung der befragten Religionswissenschaftler*innen zu einer Universität bzw. einer anderen Arbeitsstelle (Block 1) als auch ihre persönliche Einschätzungen über ihre Arbeitsbedingungen sowie beruflichen Ziele und Perspektiven erfasst (Blöcke 2 und 3). Darüber hinaus zielten Fragen auf das Erleben von Belastungssituationen und der eigenen Gesundheit (Block 4). Schließlich wurden noch einige soziodemographische Daten der teilnehmenden Personen erhoben. Darunter fielen Angaben zu Alter, Geschlecht, höchstem Bildungsabschluss und sozialen Verpflichtungen wie zum Beispiel Kindererziehung oder Pflege von Angehörigen (Block 5). Die Hauptziele waren, damit einerseits zu ermitteln, in welchen arbeitsrechtlichen Konstellationen und unter welchen weiteren Arbeitsbedingungen Religionswissenschaft vom Mittelbau und Nachwuchs betrieben wird. Auf der anderen Seite sollte festgestellt werden, von welchen Personengruppen diese Arbeit geleistet wird (Männer, Frauen, Personen mit oder ohne Kinder), ob bestimmte Gruppen auffallend häufig in bestimmten vertragsrechtlichen Konstellationen oder anderen spezifischen Arbeitskonstellationen wiederzufinden sind oder ob eine Benachteiligung aufgrund soziodemographischer Merkmale ausgemacht werden kann. Ein besonderes Anliegen war es weiterhin, der Frage nachzugehen, ob bzw. inwiefern Einflüsse der Arbeitsbedingungen auf die Gesundheit und das Privatleben (z.B. die Familienplanung) gegeben sein könnten und wie diese ggf. von den Befragten bewertet werden.

Der Erhebungszeitraum der Umfrage war der 01. bis 30. Juni 2015. Beworben wurde die Befragung im Wesentlichen über die religionswissenschaftliche Mailingliste Yggdrasill und andere Kommunikationsmedien des AKMN wie dessen Internetauftritt bei Facebook.

\section{Befunde}

\section{Die Studienteilnehmer*innen ...}

Insgesamt konnten die Datensätze von 57 Teilnehmer*innen an der Online-Erhebung verwendet werden. 28 Teilnehmer*innen gaben an, weiblich zu sein, 25 wählten die Kategorie »männlich «, drei Befragte ordneten sich der Sammelkategorie »anderes« zu, in einem Fall fehlte die Angabe. Das Alter der Teilnehmer*innen lag zwischen 22 und 50 Jahren mit einem Durchschnittswert von 32,7 Jahren. 38 Befragte hatten zum Zeitpunkt der Umfrage keine Kinder, zwölf Befragte hatten ein Kind, sieben Befragte trugen für zwei oder mehr Kinder Verantwortung.

32 Befragte ordneten sich der Gruppe der Promovierenden zu, 19 den Post-Docs bzw. Habilitierenden, die anderen gaben an, sich in einer anderen oder gar keiner Qualifikationsphase zu befinden. Diese Angaben sind konsistent mit denjenigen zum höchsten Bildungsstand der Befragten, bei dem entsprechend Magister/Master bzw. Promotion als die zuletzt erworbenen Abschlüsse angegeben wurden. Als ideales Berufsziel nennen $36,4 \%$ eine Professur oder andere Führungsposition an einer Forschungseinrichtung, 47,3\% wünschen sich eine (unbefristete) Stelle im akademischen 
Mittelbau. 16,4\% dagegen möchten am liebsten außerhalb von Hochschule und Forschungseinrichtungen arbeiten.

11 Im Vergleich mit anderen Erhebungen zeigt sich: Promovierende der Religionswissenschaft ${ }^{9}$ liegen mit 31 Jahren im allgemeinen Altersdurchschnitt aller Promovierenden, die als wissenschaftliche Mitarbeiter*innen an Universitäten tätig sind (30,6 Jahre), sie weisen mit 54,8\% gegenüber 47\% aber einen höheren Frauenanteil auf und sind seltener kinderlos (68,8\% im Gegensatz zu 85\%) (Jaksztat, Preßler und Briedis 2012, 7 und 84). Dies kann als ein erster Hinweis auf eine geringere Diskriminierung aufgrund von Geschlecht oder Familienstand in den religionswissenschaftlichen Qualifikationsverhältnissen gedeutet werden.

\section{... und ihre Beschäftigungssituation}

das Anstellungsverhältnis gilt: Zwei Drittel der Befragten sind als Wissenschaftliche Mitarbeiter*innen angestellt, etwa $10 \%$ als Lehrbeauftragte. Daneben gibt es einige vereinzelt vertretene Anstellungs- bzw. Finanzierungsmodelle, etwa als Lehrkräfte für besondere Aufgaben (LfBAs), Honorarkräfte oder Stipendiat*innen. Dieser Verteilung entspricht weitestgehend, dass $74 \%$ der Befragten befristet angestellt sind, $12 \%$ gar keinen Vertrag und 7\% einen Honorarvertrag haben. Diesen 93\% nicht oder befristet Angestellten stehen 7\% von Befragten mit einer entfristeten Anstellung oder Verbeamtung gegenüber. Die Situation des religionswissenschaftlichen Mittelbaus weist damit eine leicht höhere Befristungsquote als der Bundesdurchschnitt auf: Insgesamt sind dort $89,7 \%$ befristet und entsprechend 10,3\% unbefristet angestellt (Konsortium Bundesbericht Wissenschaftlicher Nachwuchs 2013, 16).

Sofern unter den Erhebungsteilnehmer*innen ein befristeter Vertrag vorliegt, ist dieser zu 15,4\% auf ein Jahr oder weniger, in 18\% der Fälle auf mehr als ein, aber maximal zwei Jahre, zu 51,3\% auf drei Jahre und in 15,3\% der Fälle auf mehr als drei Jahre befristet. Als Gründe für die Befristung geben 31,6\% die Projektlaufzeit eines Drittmittelprojektes und 26,2\% die sechs- bzw. zwölf-Jahresgrenze gemäß des Wissenschaftszeitvertragsgesetzes an. Bei weiteren $19 \%$ sei die Befristungsdauer von ihrem oder ihrer Vorgesetzten so festgelegt worden. Den Zahlen nach entspringt bei niemandem die Befristungsdauer dem eigenen Wunsch. Unter den $23,8 \%$ der Antworten, die weitere Befristungsgründe angeben, werden u.a. universitäre bzw. Rektoratsentscheidungen oder die Zugehörigkeit zu befristeten Professuren genannt.

Die vertragliche Arbeitszeit liegt bei der größten Gruppe $(35,2 \%)$ bei etwa 20 Wochenstunden, entspricht also etwa einer halben Stelle. Zu 10,7\% liegt sie zwischen zwei und 13 Wochenstunden. Dies umfasst möglicherweise Hilfskraftverträge, Lehraufträge, Honorarverträge oder Viertelstellen. In $7 \%$ der Fälle besteht eine vertragliche Wochenarbeitszeit von 25-34 Stunden, dabei könnte es sich also um (beispielsweise

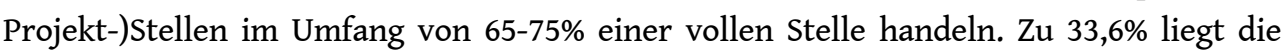
vertragliche Arbeitszeit zwischen 38 und 42 Stunden, und nimmt damit den Umfang einer vollen Stelle ein. ${ }^{10}$ Im Vergleich zur vertraglichen Arbeitszeit wurde allerdings auch die tatsächliche Arbeitszeit erhoben: Während die durchschnittliche Arbeitszeit laut Vertrag demnach bei 25,37 Stunden pro Woche liegt, liegt der Durchschnitt der Angaben zur tatsächlichen Arbeitszeit bei 32,57 Wochenstunden. Damit kann von durchschnittlich mehr als sieben Überstunden pro Woche und damit von rund 30 Überstunden pro Monat ausgegangen werden. Dies liegt damit deutlich über der bundesdeutschen 
Überstundenzahl, die 2010 bei etwa drei Stunden pro Woche (12,3 pro Monat) lag (Brautzsch und Schultz 2010). ${ }^{11}$ Gleichzeitig bleiben die Befunde im Ausmaß der unbezahlten Mehrarbeit weit hinter der ver.di-Studie zurück, die bei Inhaber*innen halber Stellen zwischen 17 und 18 Überstunden pro Woche feststellt (Grühn et al 2009, 27). Dies mag möglicherweise an der großen Zahl der Vollzeitbeschäftigten unter den befragten Religionswissenschaftler*innen liegen, die zusätzlich zu einer Vollzeitstelle kaum die gleiche hohe Zahl an Überstunden leisten (können) und auf diese Weise den Durchschnittswert beeinflussen. Trotz dieser hohen Zahl an Arbeitsstunden gibt über ein Viertel der Befragten $(27,3 \%)$ an, mit dem universitären Gehalt nicht den eigenen Lebensunterhalt bestreiten zu können (»Können Sie aus Ihrem Einkommen an der Universität Ihren Lebensunterhalt bestreiten?« »ja« oder »nein«). Als weitere Einkommensquellen werden Stipendien, externe Arbeitsverträge, Arbeitslosengeld II, Selbstständigkeit oder (mit 22,8\% die größte Gruppe) die Unterstützung durch Familie oder Partner*in genannt. Religionswissenschaftliche Arbeit wird damit zu einem signifikanten Anteil querfinanziert, sowohl aus staatlichen Mitteln als auch von Privatpersonen - und damit von verschiedenen Gruppen und Institutionen, die eigentlich nicht für die Bezahlung von Wissenschaftlicher*innen zuständig sind.

\section{(Un-) Zufriedenheit mit den Rahmenbedingungen und Perspektiven des Arbeitsverhältnisses}

15 Insgesamt zeigt sich ein erwartbares Bild: Befragt nach verschiedenen Rahmenbedingungen, liegt die größte Zufriedenheit bei allen Aspekten rund um die flexible Arbeitsorganisation vor. Basierend auf den Mittelwerten ist die Zufriedenheit mit den flexiblen Arbeitszeiten und der flexiblen Arbeitsorganisation am größten (Mittelwert von 5,4 auf einer Skala von $1=$ sehr unzufrieden bis $7=$ sehr zufrieden, Standardabweichung 1,0). Dahinter rangieren die Zufriedenheit mit den Inhalten der alltäglichen Arbeit (MW 5,3, SA 0.9), mit den Möglichkeiten, bei der Arbeit Neues zu erlernen (MW 4,9 SA 1,2), mit der Abwechslung bei der Arbeit (MW 4,9 SA 1,1) und den sozialen Beziehungen zu den Kolleg*innen (MW 4,9 SA 1,3). Die Schlusslichter bilden jene Konditionen, die mit der Sicherheit und Planbarkeit der beruflichen Laufbahn zu tun haben: Besonders unzufrieden sind die Befragten mit ihrer Vertragslaufzeit (MW 3,2 SA 1,9), ihren beruflichen Perspektiven in der Wissenschaft (MW 2,7 SA 1,5) und der Sicherheit und Planbarkeit in Bezug auf ihre Arbeitsstelle (MW 2,5 SA 1,9).

Der Erhebungsbogen gab an mehreren Stellen auch die Gelegenheit zu offenen Antworten. In Bezug auf die Zufriedenheit mit Arbeitsbedingungen deuten diese in eine ähnliche Richtung. Die Befragten sind sich hier sehr einig in ihrer Wertschätzung von Flexibilität und Eigenständigkeit bei der Arbeit. Mit großen Erwartungen an die Arbeitsleistung gehen offenbar zumindest große Freiheiten einher, was die Zeiteinteilung und die Arbeitsweisen betrifft; auch die $\mathrm{zu}$ verwaltenden Bereiche können eigenverantwortlich (mit-)gestaltet werden. Unzufriedenheit herrscht dagegen mit Blick auf zwei große Themenfelder: Die prekäre Beschäftigung sowie die Arbeitsbelastung. $\mathrm{Zu}$ Ersterem: Hierunter fallen primär die vertragliche Befristung, mangelnde Planbarkeit der Beschäftigung und unklare Berufsperspektiven. Sekundäreffekt ist aber auch das häufig beklagte Gefühl der Abhängigkeit von Vorgesetzten und der »Betonung und Ausnutzung von Hierarchien « (Zitat aus einer offenen Antwort). Dies wiederum wird in Verbindung mit dem zweiten Bereich der Arbeitsbelastung bedeutsam. Genannt werden hier die 
selbstverständlichen Erwartungen, am Wochenende zu arbeiten, jederzeit Mails zu lesen oder unbegrenzt Überstunden zu leisten. Alles drei verbindet sich insgesamt zu einem als extrem hoch erlebten Arbeits- und Qualifikationsdruck und zur teilweise geäußerten Wahrnehmung eines äußerst familienunfreundlichen Arbeitsplatzes. Ein*e Befragte* $r$ schreibt: »immer mehr zu arbeiten, als mein Vertrag es vorsieht und das auch noch gerne und selbstverständlich. Ein schlechtes Gewissen zu haben, wenn ich mal nicht so viele Überstunden mache. Themen wie Kinderwunsch oder Schwangerschaft sind im wissenschaftlichen Bereich besonders tabu. Man muss ständig um seine Stelle bangen. «12 Ein*e andere* $r$ beschreibt: "muss mich kontinuierlich wehren gegen zusätzliche Arbeitsaufgaben - also die Erwartung, dass mit einer [Teilzeit-]Projektstelle eine 100\%ige Fachkraft für die Forschungsinteressen meines/r Vorgesetzten organisiert wurde, die organisatorischen Abläufe am Lehrstuhl sind chaotisch, meine/n Vorgesetzte/n interessieren kaum die Inhalte der Forschung sondern lediglich die Einwerbung von Drittmitteln und dass sein/ihr Name überall vertreten ist.« Auch in den zuletzt erfragten allgemeinen Kommentaren zur Umfrage wird noch einmal insbesondere die hohe Leistungserwartung beklagt, mit der die Ausgrenzung aller anderen Lebenspläne oder interessen einhergehe: „Wenn man private Probleme hat, ist man schnell abgehängt. Nichts darf einen ablenken. Qualifikationen müssen schnell erbracht werden, nebenbei publizieren, zu Konferenzen fahren usw. Das klappt nur, wenn man sonst keine Verantwortungen hat.« Während sich bei den einen der Frust verfestigt (»Die bestehenden Verhältnisse ziehen Monster heran«), haben andere sich mit der Situation abgefunden: »[...] Am Anfang dachte ich, ich mache garantiert keine Überstunden, aber jetzt stellt sich diese Frage gar nicht mehr wirklich, weil Dinge einfach erledigt werden müssen. Ich gehe davon aus, dass die meisten von uns mehr arbeiten als in ihrem Vertrag steht [...].» Aussteiger*innen sehen diese Entwicklung mit Bedauern: »Ich habe Forschung und Lehre an den Universitäten geliebt, aber schnell gemerkt, wie viele hervorragende Leute im wissenschaftlichen Mittelbau kaputt gemacht werden. [...] Mir tun jene mehr Leid, die als wissenschaftliche Nachwuchskräfte kaputt gegangen oder auf ihrem Weg bitter und zynisch geworden sind. Schade, denn Religionswissenschaft ist eigentlich eine geniale und relevante Disziplin."

17 Andere Parameter aus der quantitativen Erhebung plausibilisieren wiederum diese Befunde, indem sie Aufschluss über die Einbindung in Forschung und Lehre geben. Mit Blick auf das Maß des vertraglich vereinbarten Lehrdeputats zeigt sich eine breite Streuung: Jeweils $30,2 \%$ haben kein Lehrdeputat oder bieten Lehre im Umfang von ein bis drei und weitere $39,6 \%$ von mehr als drei Semesterwochenstunden an. Dabei gilt: Wer mehr Zeit in die akademische Lehre investiert, ist mit dem Verhältnis von Arbeitszeit und Entlohnung eher unzufrieden $\left(-, 307^{*}\right)$. Dies mag daran liegen, dass die Vor- und Nachbereitung von Seminaren sowie das Durchführen der damit verbundenen Prüfungen usw. mehr Stunden kosten, als tatsächlich bezahlt werden, wie die weiteren Ergebnisse unterstreichen. Der Aufwand beträgt laut Vertrag im Durchschnitt 2,7 Stunden, die dem realen Einsatz von 3,0 Stunden gegenüberstehen. Damit ist das Maß der Lehrverpflichtung im religionswissenschaftlichen Mittelbau minimal höher, wird jedoch fairer entlohnt als im universitären Gesamtdurchschnitt, der zu 2,0 Stunden vertraglich verpflichtet ist und 2,8 leistet (Grühn et al 2009, 38). Die Höhe des Lehrdeputats steht im negativen Zusammenhang mit der Befristungsdauer des Arbeitsverhältnisses $\left(-, 372^{*}\right)$, diese Korrelation ist evtl. auf die Beschäftigung in Form von Lehraufträgen zurückzuführen, die für jeweils ein Semester vergeben werden. 

(40\%) ihrer*seiner Arbeitszeit für die wissenschaftliche Weiterqualifikation nutzen. Damit liegen die Religionswissenschaftler*innen weit hinter dem Durchschnitt aller Fachrichtungen, der in der Vorlesungszeit $40,5 \%$ und in der vorlesungsfreien Zeit sogar 49,9\% seiner Arbeitszeit für die Promotion und andere eigene Forschungsprojekte aufwenden kann (Grühn et al 2009, 37). Dabei ist diese Gelegenheit zur Weiterqualifikation eine schlüsselgröße, denn für die befragten Religionswissenschaftler*innen gilt auch: Wer in seiner Arbeitszeit nur selten an Promotion bzw. Habilitation arbeiten kann, blickt mit Enttäuschung auf die Relation zwischen seiner Qualifikation und dem aktuellen Arbeitsverhältnis $\left(-, 372^{*}\right)^{13}$, zwischen seiner Leistung und den beruflichen Chancen $\left(-, 444^{*}\right)$ sowie seiner Leistung und dem Gehalt $\left(-, 331^{*}\right)$.

Die Beurteilung der Karriereperspektiven an der Hochschule ist überwiegend pessimistisch. Während die Einschätzung der Passung von Beschäftigung und Qualifikationsbestrebung mit 74,5\% positiv ist, ebenso die Bewertung der Relation von Qualifikation und aktuellem Beschäftigungsverhältnis (91,5\%), ist die Erwartung beruflicher Chancen gemessen an individueller Leistung und Anstrengung durchwachsen: $45,1 \%$ empfinden die fehlenden Chancen als Belastung, 73,1\% sehen keine berufliche Perspektive im Hochschulbetrieb (»Ganz generell gefragt: Sind Sie der Meinung, dass Ihnen im allgemeinen Hochschul-/Wissenschaftsbetrieb eine berufliche Perspektive geboten wird?« »ja« oder »nein«). Die Zufriedenheit mit den Arbeitsbedingungen an der Universität ist mit $60,7 \%$, die zufrieden bis eher zufrieden sind, trotzdem groß (»Wie zufrieden sind Sie im Allgemeinen mit den Arbeitsbedingungen an Ihrer Universität/ Arbeitsstelle?« auf einer fünfstufigen Skala von »zufrieden« bis »unzufrieden«). Im Vergleich empfinden nur 38\% aller Sprach-, Kultur- und Sozialwissenschaftler*innen im Mittelbau ihre persönliche Arbeitssituation an der Universität als motivierend (Grühn et al 2009, 29). Es zeigt sich demnach eine große Spannung zwischen hoher Zufriedenheit mit der Arbeit bei gleichzeitigem Pessimismus und belastenden Unsicherheiten in Bezug auf die beruflichen Zukunftsperspektiven im universitären Betrieb.

\section{Belastung am Arbeitsplatz und Gesundheit}

45,5\% der Befragten berichten von keinen bis geringen Belastungen durch Zeitdruck, Überstunden und ungerechte Behandlung am Arbeitsplatz, jeweils 27,3\% leiden jedoch unter mittlerer bzw. starker bis sehr starker Belastung. Hier kann auch noch einmal auf die hohe Zahl an Überstunden verwiesen werden: Die befragten Religionswissenschaftler*innen arbeiten ein knappes Drittel ihrer regulären Wochenarbeitsstunden noch einmal unentgeltlich zusätzlich.

Hinsichtlich des Einwerbens von Drittmitteln und des Verfassens von Publikationen fühlt sich etwa die Hälfte der Befragten (52,9\%) keinerlei Druck ausgesetzt. Etwa 10\% berichten jedoch, sich einem überfordernden Publikationsdruck ausgesetzt zu fühlen, fast ein Viertel der Befragten sieht sich gezwungen, um jeden Preis Drittmittel einzuwerben. Noch drastischer ist in dieser Hinsicht wohl der Befund, dass knapp 10\% der Befragten zudem angeben, bereits durch Vorgesetzte oder Betreuer*innen zur Fälschung oder Manipulation von Forschungsergebnissen oder Daten gedrängt worden zu sein. Insgesamt haben nach den Angaben der Befragten Vorgesetzte in 13\% der Fälle ihre Rolle als Gutachter*in und in 30\% die Befristung als Druckmittel verwendet. Auch in den offenen

Zeitschrift für junge Religionswissenschaft, 11 | 2016 
Antworten werden Drohungen mit befristeten Verträgen häufiger genannt. Auffällig ist hier, dass die damit verbundenen Forderungen sich nicht nur auf eher in der Logik des Arbeitsverhältnisses liegende Bereiche wie das Fortschreiten der Dissertation oder das Einwerben von Drittmitteln beziehen, sondern auch auf die Präsenz bei Veranstaltungen jenseits der Arbeitszeit (etwa bei Abendveranstaltungen und/oder der Betreuung von Institutsgästen). Der Zusammenhang solcher Erwartungen zur möglichen Verweigerung einer Vertragsverlängerung wird, so beschreiben es mehrere Befragte, zudem recht explizit geäußert.

Die meisten Befragten beschreiben ihren Gesundheitszustand als gut (41,5\%) oder nur leicht angegriffen (47,2\%). Insgesamt ist die Mehrheit der Ansicht, dass Aspekte schlechter gesundheitlicher Verfassung einen starken $(32,9 \%)$ oder zumindest in Teilen bestehenden Zusammenhang zur Arbeit (62,5\%) aufweisen (»Wie stark schätzen Sie persönlich den Zusammenhang zwischen diesen Beschwerden und Ihrer Arbeit ein?« dreistufige Antwortskala von "starker« bis »kein Zusammenhang«). Der Rangkorrelationskoeffizient von,$- 574^{* *}$ zwischen Gesundheit und Belastung am Arbeitsplatz gibt ihnen hier Recht.

\section{Betreuung und kollegiale Rahmenbedingungen}

Als essentieller Faktor für die Zufriedenheit und Produktivität im Job gilt die Anerkennung durch Vorgesetzte und Kolleg*innen. Hier zeigt unsere Erhebung: Die Befragten sind mit den sozialen Beziehungen zu ihren Kolleg*innen etwas zufriedener (MW 4,9 von 7 SA 1,3) als mit jenen zu ihren Vorgesetzten (Mittelwert 4,7 SA 1,7, den gleichen Wert erreicht die Zufriedenheit mit flachen Hierarchien SA 1,5). Will man Vorgesetzte allerdings nicht primär an ihrer Soziabilität messen, zeigen sich etwas stärkere Unzufriedenheiten: Die Mittelwerte der Zufriedenheit mit der fachlichen bzw. organisatorischen Unterstützung durch den*die Betreuer*in liegen bei 4,2 (SA 1,6) bzw. 3,9 (SA 1,6). Der Wert für die Zufriedenheit mit der Anerkennung der eigenen Arbeit liegt bei 3,8 (SA 1,6) und damit beinahe so niedrig wie die Werte für die (Un-)Zufriedenheit mit den strukturellen Rahmenbedingungen unsicherer Berufsaussichten, die die niedrigsten insgesamt darstellen. Dabei zeigen die Daten, dass die Anerkennung auf verschiedenen Ebenen die entscheidende Schlüsselgröße darstellt: Über $61 \%$ sind zufrieden mit der Anerkennung durch ihre Betreuer*innen und Vorgesetzten, weitere 23\% sind zumindest teilweise zufrieden. Die Anerkennung korreliert mit der Aussage »Ich erhalte in schwierigen Situationen angemessene Unterstützung « (,592**), der positiven Einschätzung der Relation von Leistung und Chancen des beruflichen Fortkommens $\left(, 310^{*}\right)$, der optimistischen Bewertung der eigenen Karrieremöglichkeiten im Wissenschaftsbetrieb $\left(, 332^{*}\right)$ sowie der Belastung am Arbeitsplatz $\left(-, 445^{* *}\right)$ und dem Gesundheitszustand $\left(, 462^{* *}\right)$. Die Anerkennung der Kolleg*innen wirkt sich in ähnlicher Weise, wenn auch in etwas schwächerem Maße, positiv auf die Unterstützung in schwierigen Situationen $\left(, 352^{* *}\right)$ und die Einschätzung der beruflichen Chancen $\left(, 289^{*}\right)$ aus.

Wenn $74,5 \%$ der Befragten mit den sozialen Beziehungen zu ihren Vorgesetzten und $79,9 \%$ mit der Beziehung zu ihren Kolleg*innen eher bis sehr zufrieden sind, bedeutet dies auch, dass ein Viertel bzw. ein Fünftel der Befragten mit Vorgesetzten bzw. kollegialer Zusammenarbeit eher oder sehr unzufrieden sind. Dementsprechende Schilderungen finden sich auch in den offenen Fragen, etwa von schlechten 
Führungskompetenzen der Vorgesetzten oder strategischer Denunziation unter Kolleg*innen. Diese Antworten erhellen entsprechend die Gründe für spezifische Unzufriedenheit und weisen zum Teil auf negative Sekundäreffekte dieser Konstellationen hin, etwa die allgemeine Schmälerung von Produktivität durch die resultierende hohe Fluktuation von Mitarbeiter*innen. Den offenen Antworten zu entnehmen sind weiterhin Aufgaben, die aus Sicht der Befragten von ihnen selbst erledigt werden müssen, obwohl sie eigentlich von ihrer oder ihrem Vorgesetzten zu übernehmen wären. Die größte Rolle spielt hier die Übernahme des Lehrbetriebs, etwa bei der Übernahme von Lehrverpflichtungen der Vorgesetzten oder der Abnahme von Prüfungsleistungen und Studierendenbetreuung in unangemessenem Maße. Andere Punkte betreffen die Repräsentation des Studienganges in öffentlichen Veranstaltungen und die Vertretung der Vorgesetzten in Sitzungen, Drittmittelverwaltung und anderen die Vorgesetzten betreffende Verwaltungsarbeiten. Auch das Verfassen von Drittmittelanträgen im Namen der Vorgesetzten wird genannt, was aber mit Vorgaben seitens der Drittmittelgeber zumindest in Teilen zu erklären sein mag. Nicht als Problem benannt wird dagegen das in anderen Disziplinen wohl üblichere Verfassen von Publikationen unter dem Namen der Vorgesetzten.

\section{Geschlecht und Kinder(wunsch)}

25 Alle im Rahmen der Ergebnisdarstellung dieses Artikels untersuchten Variablen variieren unabhängig vom Geschlecht, dies kann als Indikator für eine weitgehend geschlechtsneutrale Fachkultur gewertet werden. Alle analysierten Variablen sind in ihren Ausprägungen weiterhin unabhängig vom Vorhandensein von Kindern. Daraus kann zunächst geschlossen werden, dass keine systematische Diskriminierung von Personen, die Kinder haben, stattfindet. Keinen Aufschluss gibt die Erhebung allerdings darüber, inwiefern Elternschaft arbeitgeberseitig als Einstellungshindernis betrachtet wird oder bereits arbeitnehmerseitig dazu führt, dass sich Eltern gegen eine möglicherweise als familienunfreundlich empfundene akademische Karriere entscheiden.

32,1\% der Befragten haben ein oder mehrere Kinder, und dies ist unabhängig von Qualifizierungsphase, arbeitsvertraglichem Verhältnis oder vergleichbaren Variablen. Sowohl unter den promovierenden Religionswissenschaftler*innen als auch in der Gesamtstichprobe ist Elternschaft weiterhin unabhängig vom Geschlecht. Die HIS-Studie hingegen weist für den Gesamtdurchschnitt der Promovierenden, die in Forschungsprojekten oder an Lehrstühlen arbeiten, einen signifikanten Geschlechterunterschied nach. Dieser besteht darin, dass unter denjenigen, die ein Kind unter drei Jahren haben, die Männer leicht überrepräsentiert sind, während unter denjenigen, die ein Kind über drei Jahren haben, die Frauen leicht überrepräsentiert sind (Jaksztat, Preßler und Briedis 2012, 84). Immerhin 44\% der befragten Religionswissenschaftler*innen - und darunter in gleichem Maße Männer und Frauen geben allerdings an, einen Kinderwunsch aufgrund der akademischen Laufbahn zurückzustellen (»Haben Sie schon einmal einen Kinderwunsch aufgrund Ihrer akademischen Laufbahn zurückgestellt?« »ja« oder »nein«). Als Gründe werden von den allermeisten Befragten (rund 80\%) die fehlende planerische Sicherheit und von rund der Hälfte die Unvereinbarkeit von Familie und Beruf aufgrund ihrer Arbeitszeiten und fehlender Betreuungsmöglichkeiten angeführt. Gut 30\% benennen aber weiterhin oder ausschließlich entsprechenden Druck durch ihre*n Vorgesetzt*e als ausschlaggebenden 
Faktor. Es lässt sich damit folgern, dass sich statistisch keine Diskriminierung aufgrund von Geschlecht oder Elternschaft zeigt. Angesichts der hohen Zahl zurückgestellter Kinderwünsche liegt auch die Hypothese einer gelungenen Adaption nahe: Möglicherweise wagen gerade die Befragten in Arbeitsverhältnissen, in denen ein kinderunfreundliches Klima herrscht, gar nicht erst den Schritt zur Elternschaft, so dass hier folgerichtigerweise auch keine Diskriminierung von Beschäftigten mit Kindern erfolgt.

\section{Zusammenfassung und Ausblick}

Einigermaßen erwartbar stellten sich Zufriedenheiten und Unzufriedenheiten mit einzelnen Aspekten der Arbeitstätigkeit dar. Dabei lässt sich festhalten, dass jene strukturellen Bedingungen, die über das direkte Arbeitsumfeld hinausgehen, jeweils Extrempositionen hinsichtlich Zufriedenheit und Unzufriedenheit einnehmen: Besonders beklagt wird die große berufliche Unsicherheit und alles damit Einhergehende, besonders gelobt dagegen die große Flexibilität bei der konkreten Arbeit. Diese Extrempositionen mögen auch darin begründet liegen, dass diese Aspekte in Teilen für universitäre Beschäftigungsverhältnisse im Allgemeinen gelten und demnach für alle Befragten etwa gleiche Bedingungen herrschen, wohingegen die (Un-)Zufriedenheiten im kleineren Umfeld von Instituten und Beziehungen zu Betreuer*innen und Vorgesetzten jeweils singulären Konstellationen entspringen. Gerade die Zufriedenheit mit der Beziehung zu Vorgesetzten etwa zeigt dementsprechend auch eine hohe Streuung.

Als ein wichtiger Faktor stellte sich dabei jener der Anerkennung durch Vorgesetzte oder Betreuer*innen dar. Angesichts der Relevanz dieses Faktors, auch hinsichtlich der Korrelation mit anderen Aspekten, scheint es erstaunlich, dass hier ein so großer Mangel beklagt wird - zumal die Anerkennung gleichsam »billig zu haben ist«, indem sie nicht etwa gegen die Widrigkeiten der Systemlogiken erkauft werden müsste (wie es etwa bei längeren Vertragslaufzeiten der Fall sein könnte). Auch wenn wir keine Aussagen über Kausalitäten treffen können, springen doch die hohen Korrelationen mit dem Gesundheitszustand der Befragten und dem Gefühl von Überlastung am Arbeitsplatz ins Auge. Erschreckend erscheint uns weiterhin der Druck, der laut den statistischen Angaben sowie den Beschreibungen in den offenen Antworten von Vorgesetzten ausgeübt wird. Hierbei muss betont werden, dass es sich um Einzelfälle, nicht um den Regelfall handelt. Dennoch ist dieser Befund alarmierend, da der genannte Druck offenbar sogar bis zu der Erwartung reicht, Forschungsergebnisse zu fälschen.

Einen erfreulichen Befund stellt die weitgehend egalitäre bzw. diskriminierungsfreie Kultur mit Blick auf Geschlecht und Elternschaft dar, die sich positiv von der anderer Fächer abhebt. Dennoch kann die hohe Zahl derjenigen, die einen Kinderwunsch aufgrund der unsicheren Arbeitsbedingungen oder der ablehnenden Haltung von Betreuer*innen oder Vorgesetzten verschieben, nachdenklich stimmen und auch eine gelungene Adaption der Mitarbeiter*innen an die Erwartungen ihrer Vorgesetzten vermuten lassen. Dessen unbenommen: Auch wenn es im akademischen Umfeld üblich ist, sich über die Maße für seinen Beruf zu engagieren, erscheint es doch wenig wünschenswert, dass private Lebensplanung in solch hohem Maße dahinter zurücksteht insbesondere wenn bedacht wird, dass der langfristige Verbleib im akademischen Umfeld realistischer Weise nur für einen Bruchteil der Befragten möglich sein wird. Während die unsicheren Arbeitsbedingungen in weiten Teilen sicherlich ein systemisches Problem 
darstellen, liegt zumindest eine familienfreundliche Haltung im konkreten Arbeitsumfeld in den Händen der Betreuer*innen und Vorgesetzten und ist gleichermaßen nicht mit allzu hohen, schon gar nicht in gleichem Maße persönlichen, Opportunitätskosten verbunden. Eine durchweg unterstützende - oder zumindest gleichgültige! - Haltung der Vorgesetzten und Betreuer*innen hinsichtlich der Familienverhältnisse ihrer Mitarbeiter*innen wäre daher ebenso wünschenswert wie sie uns naheliegend erscheint.

Auffällig ist weiterhin die hohe Zahl an Überstunden. Teilzeitstellen machen diese hohe absolute Zahl an Überstunden erst möglich, sie werden dadurch aber noch unverhältnismäßiger zur bezahlten Gesamtarbeitszeit. Hier ist sicherlich in Rechnung zu stellen, dass viele religionswissenschaftliche Einrichtungen institutionell bereits gewissermaßen rauf Kante genäht sind, und den Alltag von Lehre und Studienorganisation nur durch ein überdurchschnittliches Engagement aller Beteiligten stemmen können. Gleichzeitig resultieren diese Überstunden für den wissenschaftlichen Nachwuchs de facto auch in weniger Zeit für die wissenschaftliche Weiterqualifikation im Vergleich $\mathrm{zu}$ anderen Fächern. Hier ist zu bedenken, dass religionswissenschaftliche Promovierende und Post-Docs in vielen - auch akademischen - Berufsfeldern in direkter Konkurrenz mit Regionalwissenschaftler*innen, Philolog*innen, Sozial- und Kulturwissenschaftler*innen stehen, so dass es in niemandes Interesse sein kann, dem eigenen Nachwuchs im Vergleich geringere Qualifikationsgelegenheiten zu schaffen.

Ein letzter Aspekt betrifft erneut eine Melange aus systembedingten und konkreten Arbeitsbedingungen: Die Erhebung zeigte eine erfreulich hohe Anzahl an Vollzeitstellen, doch auch eine nicht unerhebliche Zahl von sehr kurzen Befristungen. Diese Stellen mit Laufzeiten von maximal zwei Jahren lassen offen, inwieweit dies mit Projektlaufzeiten erklärt werden kann, vor allem aber, wie dies zu dem Umstand passt, dass es sich hierbei zumindest in Teilen auch um Qualifikationsstellen handeln dürfte. Auch angesichts der im Fächervergleich insgesamt hohen Anzahl an befristeten Stellen, die häufig mit dem Wissenschaftszeitvertragsgesetz oder der Festlegung des oder der Vorgesetzten begründet wird, sei nur pro forma noch einmal daran erinnert, dass das Wissenschaftszeitvertragsgesetz nicht die Weiterbeschäftigung von Mitarbeiter*innen nach sechs bzw. zwölf Jahren verbietet. Die Weiterbeschäftigung ist durchaus möglich aber eben entfristet.

Dabei ist offenkundig, dass die gegenläufige Perspektive des sechs-Jahre-hire-and-fire ebenso wie viele andere hier thematisierte Problemlagen der Arbeitsverhältnisse für Mittelbau und Nachwuchs - etwa die fehlende Zeit zur individuellen Weiterqualifikation, die hohe Arbeitsbelastung, die sich auch auf Gesundheitszustand und Familienplanung der Befragten auswirkt - im universitätsweiten Usus ihren Ausgangspunkt finden. Der unserer Erhebung zugrunde liegende Fokus auf bestimmte Statusgruppen, der sowohl Professor*innen als auch Studierende ausschließt, soll keinesfalls suggerieren, dass die aufgezeigten Probleme diese nicht betreffen. Vielmehr ist offenkundig, dass permanenter Zeitdruck sowie hohe Erwartungen an die Zahl der Publikationen und den Umfang der eingeworbenen Drittmittel auch andere Statusgruppen, insbesondere auch Professor*innen treffen, in ihrem derzeitigen Arbeitsumfeld ebenso wie im vorangegangenen Karriereverlauf. ${ }^{14}$ Doch auch wenn viele Schwierigkeiten des akademischen Arbeitsumfeldes nicht in Vorgesetzten oder Instituten begründet liegen, ließe sich doch möglicherweise darüber nachdenken, welche Systemlogiken man instituts- oder lehrstuhlintern mittragen und damit gewissermaßen snach unten durchreichen will, und welchen man sich entziehen oder aber als Vorgesetzte*r bzw. 
gemeinsam entgegenstellen kann. Dies beginnt bei den Parametern der Anstellung: Mit der diesjährigen Änderung des Wissenschaftszeitvertragsgesetz werden unsachgemäße Kurzzeitbefristungen bereits erschwert, und eine Mindestbefristung auf die betreffende Projektlaufzeit oder angemessene Qualifizierungszeiten von fünf bis sechs Jahren bei gleichzeitiger Einbindung in Lehre und Gremienarbeit erscheinen absolut naheliegend. Für Fälle von grundsätzlicher Unzufriedenheit mit Mitarbeiter*innen gilt ohnehin die gesetzlich geregelte Probezeit, die Möglichkeit allerdings, mit jährlich zu verlängernden Verträgen Druck auszuüben, entfiele damit. Immer noch scheint es üblich, Mitarbeiter*innen auf halben Stellen zu beschäftigen. Hier sei daran erinnert dass bereits der DFG-Regelsatz für Promotionsstellen in der Religionswissenschaft bei $75 \%$ liegt. Sofern Professuren ausschließlich eine halbe Mitarbeiterstelle zugeordnet ist, bleiben solche Aufstockungen natürlich Wunschdenken. Nicht unüblich erscheint allerdings auch, eine ganze Stelle oder ein größeres Stellenpaket in möglichst viele halbe Stellen aufzuteilen, ein Usus, der die bereits angesprochene Querfinanzierung religionswissenschaftlicher Arbeit, notwendige Weiterqualifikation in der Freizeit sowie selbstverständliche Überstunden beinahe automatisch mit sich führt. Gerade die Qualifikation als Religionswissenschaftler*in ist zudem als Teil der Arbeitsleistung zu verstehen, und nicht vollständig in die unbezahlte Mehrarbeit auszulagern. Sie ist essenzieller Teil der konkret erfolgenden religionswissenschaftlichen Forschung (wie sich u.a. auf religionswissenschaftlichen Konferenzen an dem hohen Anteil der Vortragenden aus dem Mittelbau zeigt); sie fließt im Idealfall in die Lehre ein, und erweitert das Fachwissen aller an diesem Prozess Beteiligten. Mitnichten ist sie daher Privatvergnügen der betroffenen universitär angestellten Religionswissenschaftler*innen.

Privatvergnügen sind zuletzt gewissermaßen Familie und Gesundheit. Solcherlei Luxus kann sich aber nur leisten, wer über die Freiheit hinreichenden Privatlebens verfügt. Daher sei noch einmal recht unsensibel daran erinnert, dass die universitäre Anstellung der Mittelbauler*innen im Durchschnitt kurz, das Leben aber im Idealfall lang ist - umso naheliegender erscheint uns, gemeinsam an fruchtbaren, ergiebigen Arbeitsverhältnissen ebenso zu arbeiten wie ein rekreatives Privatleben zu ermöglichen.

Abschließend sind mit Blick auf die Aussagekraft der vorliegenden Studie einige Einschränkungen festzuhalten. So konnte insbesondere die Situation der außeruniversitär religionswissenschaftlich Forschenden nicht ausreichend berücksichtigt werden. Auch wenn die Erhebung sie prinzipiell miteinbeziehen sollte, waren große Fragebereiche für diese Gruppe irrelevant. Zudem lassen die Daten kaum valide Unterscheidungen zwischen den Gruppen der Angestellten und der Nicht-Angestellten zu. Eine aktualisierte Fassung des Fragebogens könnte solche institutionellen Anstellungsverhältnisse differenzierter berücksichtigen.

Weiterhin ist die erreichte Stichprobengröße für die Untersuchung einer Reihe von Zusammenhängen, etwa zwischen Geschlecht und Status innerhalb des Mittelbaus, zu gering. Dies lässt sich wohl nicht mit einer erneuten Erhebung optimieren, sondern liegt auch in einer zahlenmäßig geringen Grundgesamtheit begründet. Aufgrund unterschiedlicher nationaler Universitätsstrukturen erscheint allerdings auch eine Erweiterung etwa auf den europäischen Raum wenig sinnvoll. ${ }^{15}$ Eine naheliegendere Annäherung stellt hingegen der hier auch thematisierte Vergleich mit disziplinübergreifenden Erhebungen dar. Eine weitere aufschlussreiche Ergänzung könnte in der Erhebung qualitativer Daten bestehen, die solchen hier kaum zu postulierenden statistischen Korrelationen einen tiefergehenden Einblick in 
zugrundeliegende Logiken und Muster zwischen Charakteristika der Befragten, ihren Anstellungsverhältnissen und deren Einschätzung zur Seite stellt. Zuletzt fehlt eine kontrollierte Stichprobenziehung, um die Repräsentativität und Generalisierbarkeit der Ergebnisse zu gewährleisten - auch dies ein Sachverhalt, dem aufgrund der ausgesprochen kleinen und heterogenen Grundgesamtheit kaum mit weiteren statistischen Erhebungen beizukommen ist.

Die angeführten Daten und Interpretationen können daher nur einen ersten Eindruck vom thematisierten Feld geben. Auch andere - etwa studentische oder professorale Perspektiven auf den Sachverhalt könnten nun aufschlussreich sein. Wir würden uns daher vor allem freuen, mit diesem Beitrag eine weiterführende Debatte angeregt $\mathrm{zu}$ haben.

\section{BIBLIOGRAPHY}

Brautzsch, Hans-Ulrich, und Birgit Schultz. 2012. IWH-Pressemitteilung 34/2012. Halle (Saale): Institut für Wirtschaftsforschung Halle.

Grühn, Dieter, Hecht, Heidemarie, Rubelt, Jürgen, und Boris Schmidt. 2009. Der wissenschaftliche "Mittelbau« an deutschen Hochschulen. Zwischen Karriereaussichten und Abbruchtendenzen. Berlin: ver.di.

Internationale Expertenkommission Exzellenzinitiative. 2016. Endbericht. Berlin: Institut für Innovation und Technik (iit).

Jaksztat, Steffen, Preßler, Nora, und Kolja Briedis. 2012. Promotionen im Fokus. Promotions- und Arbeitsbedingungen im Vergleich. Hannover: HIS Hochschul-Informations-System.

Konsortium Bundesbericht Wissenschaftlicher Nachwuchs. 2013. Bundesbericht Wissenschaftlicher Nachwuchs. Bielefeld: Bertelsmann Verlag.

Kreckel, Reinhardt. 2012. »Habilitation versus Tenure. Karrieremodelle an Universitäten im internationalen Vergleich.« Forschung \& Lehre 1: 12-15.

Krüger, Oliver. 2016. »Religionswissenschaft als Beruf. Probleme und Perspektiven der universitären Karriere.« Zeitschrift für Religionswissenschaft 24 (2): 205-212.

Mittelbauinitiative Universität Leipzig (MULE). 2015. Umfrage Mittelbau Universität Leipzig. Letzter Zugriff: 11. August 2016.

https://notraces.net/limesurvey/index.php/775175/lang-de

Pürschel, Tom, und Jana Rüger. 2016. »Akademischer Mittelbau als Beruf. Bericht aus dem Kuriositätenkabinett.« INDES. Zeitschrift für Politik und Gesellschaft 1. Letzter Zugriff: 11. August 2016.

indes-online.de/1-2016-akademischer-mittelbau-als-beruf

Weber, Max. 1992 [1917/1919]. Wissenschaft als Beruf. Hrsg. von Wolfgang J. Mommsen, und Wolfgang Schluchter. Tübingen: J.C.B. Mohr (Paul Siebeck). 


\section{NOTES}

1. Vgl. für einen Kommentar dazu Krüger 2016.

2. Die Auswertung basierte auf 600 vollständig ausgefüllten Fragebögen von wissenschaftlichen Mitarbeiter*innen, die an einer von drei ausgewählten deutschen Universitäten befristet angestellt waren.

3. In der Stichprobe von 2.850 Datensätzen waren frei Promovierende, Teilnehmer*innen von Stipendien- und strukturierten Promotionsprogrammen sowie wissenschaftliche Mitarbeiter*innen in universitären Forschungsprojekten, an Lehrstühlen und außeruniversitären Forschungseinrichtungen vertreten.

4. Der Bericht bezieht seine Daten, die ausschließlich Bezug auf die Bundesebene nehmen, aus verschiedenen Quellen amtlicher Statistiken und trägt eine Vielzahl einschlägiger Forschungsergebnisse zusammen.

5. Es kann nicht ausgeschlossen werden, dass in geringem Umfang deutschsprachige Religionswissenschaftler*innen, die außerhalb Deutschlands arbeiten, promovieren oder sich habilitieren etc. an der im folgenden vorgestellten Umfrage teilgenommen haben. Die Handlungsempfehlungen in diesem Beitrag beziehen sich jedoch in erster Linie auf religionswissenschaftliche Lehr- und Forschungseinrichtungen an Universitäten in Deutschland. Zur Beschreibung der Teilnehmenden und Reichweite der Untersuchung siehe die Abschnitte 3.1 und 7.

6. Allen Beteiligten an den vorangehenden Diskussionen sowie allen Teilnehmenden möchten wir daher herzlich danken.

7. Angesichts der Datenlage ist dies nur für einzelne Items möglich. Es erscheint uns daher kaum legitim, arbeitsfeldübergreifende Aussagen $\mathrm{zu}$ treffen, etwa, inwiefern sich die Religionswissenschaft mit Blick auf (Un-)Zufriedenheit mit den Rahmenbedingungen des Arbeitsverhältnisses oder subjektiv empfundene Belastung von anderen Fächern oder Arbeitsfeldern in positiver oder negativer Weise abhebt. Einige Anhaltspunkte können aber die im Folgenden aufgeführten, punktuell möglichen disziplinübergreifenden Vergleiche, etwa zu Geschlechterverhältnissen und Familienstand unter Promovierenden, liefern.

8. Vgl. auch: Pürschel und Rüger 2016.

9. Aus der Gesamtstichprobe wurden anhand der Variable »In welcher Qualifizierungsphase befinden Sie sich derzeit?« die Promovierenden ausgewählt.

10. Die fehlenden Prozentwerte sind auf fehlende Angaben in den Fragebögen zurückzuführen.

11. Hierbei muss bedacht werden, dass im Bundesdurchschnitt mehr als die Hälfte der Überstunden vergütet wird; eine Praxis, die im universitären Arbeitsfeld ungewöhnlich sein dürfte. Weiterhin ist darauf hinzuweisen, dass es sich bei den hier untersuchten Beschäftigungsverhältnissen mehrheitlich um Teilzeitbeschäftigungen handelt, die Überstundenzahl in Relation zur vertraglich geregelten Arbeitszeit also möglicherweise noch stärker ins Gewicht fällt.

12. Die Zitate sind wörtlich der Umfrage entnommen, nur eindeutige Tippfehler wurden korrigiert. An manchen Stellen wurden die Antworten weitergehend anonymisiert, etwa, indem genaue Angaben zu Stellenumfang oder spezifischen Arbeitsaufgaben entfernt wurden. Diese Veränderungen sind mit eckigen Klammern gekennzeichnet.

13. Bei allen Korrelationskoeffizienten in der Darstellung der Ergebnisse handelt sich um Rangkorrelationskoeffizienten nach Spearman mit einem zweiseitigem Test der Signifikanz, ${ }^{*} \mathrm{p}<0,05 .{ }^{* *} \mathrm{p}<0,01$.

14. Man könnte daher argumentieren, dass der Umgang mit diesen Anforderungen einen notwendigen Lernprozess auf dem Weg zu möglichen Führungsaufgaben darstellt. Unsere 
Prämisse für die folgenden Ausführungen ist gleichwohl, dass ein als überfordernd empfundener Zeitdruck für keine der Statusgruppen, die Wissenschaft betreiben, einen wünschenswerten Zustand darstellt.

15. In Bezug auf die Universitätssysteme anderer Länder wären gleichwohl aufschlussreiche Kontrastierungen denkbar. So hat Reinhard Kreckel mit einer vielbeachteten Zusammenstellung deutlich vor Augen geführt, dass "allein Deutschland über ein Universitätssystem verfügt, in dem fest angestellte, eigenständig lehrende und forschende Hochschullehrer beim hauptberuflichen wissenschaftlichen Personal klar in der Minderheit sind« (Kreckel 2012, 12). Mit einer geeigneten Datenbasis ließe sich daher sicherlich - dann aber wohl disziplinenübergreifend - genauer in den Blick nehmen, welche Probleme beispielsweise ein stärker tenure-basiertes Universitätssystem bereits löst, und welche Sachverhalte wiederum andere Lösungen erfordern würden.

\section{ABSTRACTS}

Die Arbeitsbedingungen und -zufriedenheit des religionswissenschaftlichen Nachwuchses, d.h. all derer, die ohne auf eine Professur berufen zu sein, religionswissenschaftlich forschen und arbeiten, wurden bislang kaum untersucht. Der Arbeitskreis Mittelbau und Nachwuchs (AKMN) der Deutschen Vereinigung für Religionswissenschaft (DVRW) hat im Sommer 2015 eine statistische Erhebung durchgeführt, um diese Lücke zu schließen. Auf der Grundlage der Umfrageergebnisse werden in diesem Beitrag zunächst die Arbeitsbedingungen und ihre Auswirkungen auf berufliche Perspektiven und persönliche Lebensumstände der betreffenden Personengruppe beschrieben. An diese Darstellung schließen sich Anregungen für die zukünftige Gestaltung religionswissenschaftlicher Arbeit im Kontext von Forschungsinstitutionen, insbesondere Universitäten, an.

The working conditions of young researchers of the study of religions have not been studied in depth. In order to fill up this gap, the working group of young researchers within the German association for the study of religions carried out a survey in summer 2015. Based on the survey results this paper describes the working conditions and their effects on the professional perspectives and the individual life situations of the group in question. After presenting such findings, we conclude with an outlook and with several suggestions in regard to the possible future design of the work in the study of religions in the context of research institutions, and especially at universities in Germany, focussing specifically on appreciation of work, pressure within the relation to supervisors, discrimination, workload and the opportunity of ongoing scientific qualification.

\section{INDEX}

Keywords: Mid-level Faculty, Young Scholarship, Working Conditions, Study of Religion Schlüsselwörter: Mittelbau, Junge Forschung, Arbeitsbedingungen, Religionswissenschaft 


\section{AUTHORS}

\section{VERONIKA EUFINGER}

Veronika Eufinger studierte an der Ruhr-Universität Bochum Psychologie, Philosophie und Religionswissenschaft. Sie arbeitete bis 2015 an der Evangelischen Hochschule RheinlandWestfalen-Lippe im Forschungsprojekt »Auf dem Weg in eine säkulare Gesellschaft? Kirchlichkeit, Religiosität und religiöse Weltsicht in Geschlechterperspektive.« Zurzeit ist sie Doktorandin am Centrum für Religionswissenschaftliche Studien (CERES) und Wissenschaftliche Mitarbeiterin am Zentrum für angewandte Pastoralforschung (ZAP). Ihre Forschungsinteressen liegen in den Bereichen Religion im urbanen Raum sowie Religion und Geschlecht aus der Perspektive quantitativer und qualitativer Sozialforschung.

Kontakt: Veronika.Eufinger@ruhr-uni-bochum.de

\section{RAMONA JELINEK-MENKE}

Ramona Jelinek-Menke studierte Religionswissenschaft und Soziologie an den Universitäten Göttingen und Leipzig. Nach dem Studium arbeitete sie von 2014 bis 2015 als wissenschaftliche Koordinatorin am Centre for the Study of Religion an der Universität Leipzig (CSR). Derzeit ist sie Doktorandin am Religionswissenschaftlichen Seminar der Universität Zürich und forscht zum Thema Religion and Dis/ability. Ihre Forschungsinteressen sind außerdem die Zusammenhänge von Religionen und Wohlfahrtspflege, das Verhältnis zwischen Religionen und Eugenik sowie die interpretative Sozialforschung und Biographieforschung in der Religionswissenschaft.

Kontakt: Ramona.Jelinek-Menke@uzh.ch

\section{ANNA NEUMAIER}

Dr. Anna Neumaier studierte Kultur-, Religions- und Musikwissenschaften an den Universitäten Bremen und Leipzig. Bis 2014 war sie wissenschaftliche Mitarbeiterin am Centrum für Religionswissenschaftliche Studien (CERES) der Ruhr-Universität Bochum und wurde dort mit einer Arbeit zu religionsbezogener Internetnutzung promoviert. Derzeit ist sie wissenschaftliche Mitarbeiterin im Fachgebiet „Empirische Religionsforschung und Theorie der Religion« am Institut für Religionswissenschaft und -pädagogik der Universität Bremen sowie Mitarbeiterin im DFG-Projekt »Identität angesichts religiöser Pluralität«. Weitere Forschungsinteressen gelten qualitativer Religionsforschung, religiöser Gegenwartskultur sowie Religion und neuen Medien. Kontakt: Neumaier@uni-bremen.de 